\title{
Lead Iodide Crystals as Input Material for Radiation Detectors
}

\author{
Sunil Kumar Chaudhary \\ Department of Physics, Maharshi Dayanand University, Rohtak, India \\ Email: sunilkc2001in@yahoo.com
}

Received September 3, 2012; revised October 6, 2012; accepted October 15, 2012

\begin{abstract}
Lead iodide is an important inorganic solid for fundamental research and possible technological applications and is considered to be a potential room temperature nuclear radiation detector. In lead iodide the phenomenon of polytypism is posing an interesting problem of phase transformations amongst its various polytypic modifications. The transformations have also been observed even when the crystals are stored for few months. It causes deterioration in functioning of $\mathrm{PbI}_{2}$ devices. Taking into account the known structures of $\mathrm{PbI}_{2}$ and the data available on the mode of growth and storage of crystals, it has been concluded that purified melt grown crystals of $\mathrm{PbI}_{2}$ are the best suited for nuclear radiation detectors.
\end{abstract}

Keywords: Lead Iodide; Polytypism; Crystal Structure; Phase Transformations

\section{Introduction}

In the last decade the technology of radiation detectors has improved greatly. Room temperature operated detectors have found many applications such as image devices, preventing nuclear matter smuggling and scientific instruments. The lead iodide and mercuric iodide are the leading candidates because of wide band gap that makes high resistivity, large mass density and high atomic number that provides a high stopping power [1]. Out of these the lead iodide has an edge over mercuric iodide because growing single crystals of lead iodide are simpler as compared to mercuric iodide as lead iodide has lower vapour pressure than mercuric iodide and better chemical stability. Further, phase transition between orthorhombic and tetragonal phases of mercury iodide at $400 \mathrm{~K}$ limits the bulk growth of mercuric iodide crystals and can be grown only by vapour phase.

Polytypism has been engaging interest of scientists for long by the fact that various structures of the same substance have been found to possess different electronic properties [2]. In the case of lead iodide the co-existence of more than one polytype in the same crystal, occurrence of phase transformations during storage, transformations to various modifications due to presence of native impurities and defects are some of the effects that may cause notable deterioration of their performance as detector. Polytypic effects can be serious in electrical power applications utilizing very wide band gap crystal- line material $\mathrm{SiC}$ [3] and such problems have also been frequently encountered in pharmaceutical applications and patients intake safety [4]. There are number of technological processes like average energy per electron-hole pair, typical mean time for the carriers mu-tau products, charge collection efficiency and the resistivity required to be optimized for the fabrication of $\mathrm{PbI}_{2}$ detectors but initially it is necessary to grow the pure crystals that may be required for device application with reproducible properties. To achieve this it becomes obvious to study their mode of growth and phase transformations on storage.

Recently Matuchova et al. [5] have investigated $\mathrm{PbI}_{2}$ crystals for their conductivity with and without impurities. Crystals prepared after purification with 20 zone passes, the conductivity was reduced to $10^{-13} \Omega^{-1} \mathrm{~cm}^{-1}$. Further a decrease in conductivity was also observed amongst the crystals synthesized in the presence of Ho and $\mathrm{Tm}$ (rare earth elements) but no satisfactory explanation has been given. A deep understanding of role played by the impurities can be helpful in optimizing the conditions of growth of crystals for the fabrication of desired quality of the detector.

Therefore considering the known structures of $\mathrm{PbI}_{2}$ from Inorganic Crystallographic Structure Database (ICSD) and the mechanism of phase transformations in lead iodide due to distortion of $\left[\mathrm{PbI}_{6}\right]^{4-}$ octahedron, we review the effect of storage in the crystals grown by various different modes and conclude for the most suitable method for the device application. 


\section{Known Polytypes and Phase Transformations in Lead Iodide Crystals}

The lead iodide crystals can be prepared by various methods but prominently these are grown by gel, vapour and melt. It crystallizes in Hexagonal and rhombohedral structures and till today more than 40 polytypes have been reported and a total of 20 structures have been determined. Table 1 shows the known structures of $\mathrm{PbI}_{2}$. It is clear that main basic types of crystalline $\mathrm{PbI}_{2}$ polytypic modifications are $2 \mathrm{H}, 4 \mathrm{H}$ and $12 \mathrm{R}$. Here $\theta$ characterizes the departure from ideal octahedron $\left[\mathrm{PbI}_{6}\right]^{4-}$. It is to be noted that each polytype has different departure from octahedral $\left[\mathrm{PbI}_{6}\right]^{4-}$ arrangement. One such distorted octahedron is shown in Figure 1. Departure from exact octahedron (all values of $\theta$ are zero for a perfect octahedron) may be due to presence of impurities in the starting material. It is worth-noting that all the crystals whose structures were determined have been grown from vapour or gel. From 1959 to 1985 over a period of 25 years there is no discussion of impurities that might be responsible for departure from octahedral arrangement. It is well established that polytype $2 \mathrm{H}$ is stable when crystals are grown at room temperature and $12 \mathrm{R}$ is stable when the crystals are grown at high temperature [6]. $4 \mathrm{H}$ is considered to be a metastable structure that is formed when a $2 \mathrm{H}$ structure is annealed at a temperature over $140^{\circ} \mathrm{C}-200^{\circ} \mathrm{C}$ [7]. It has also been reported that laser irradiation of $2 \mathrm{H}$ polytype of $\mathrm{PbI}_{2}$ cannot produce $2 \mathrm{H} \rightarrow$ $4 \mathrm{H}$ phase transformation but can induce irreversible $4 \mathrm{H} \rightarrow$ $2 \mathrm{H}$ phase transformation [8].

Table 1. $\mathrm{PbI}_{2}$ polytypes with known structures.

\begin{tabular}{|c|c|c|c|c|c|c|}
\hline ICSD $^{\#}$ & $\mathrm{~Pb}-\mathrm{I}(\mathrm{nm})$ & $\theta\left({ }^{\circ}\right)$ & $\mathrm{Z}$ & poly-type & $\mathrm{a} \& \mathrm{~b}(\mathrm{~nm})$ & $\mathrm{c} / \mathrm{Z}(\mathrm{nm})$ \\
\hline 024262 & 0.322 & 0.22 & 1 & $2 \mathrm{H}$ & 0.4557 & 0.6979 \\
\hline 042013 & 0.323 & 0.21 & 1 & $2 \mathrm{H}$ & 0.4557 & 0.6979 \\
\hline 068819 & 0.323 & 0.16 & 1 & $2 \mathrm{H}$ & 0.4558 & 0.6986 \\
\hline 024263 & 0.322 & 0.20 & 2 & $4 \mathrm{H}$ & 0.4557 & 0.6979 \\
\hline 024265 & 0.322 & 0.20 & 3 & $6 \mathrm{H}$ & 0.4557 & 0.6979 \\
\hline 024264 & 0.321 & 0.40 & 3 & $6 \mathrm{R}$ & 0.4557 & 0.6979 \\
\hline 060327 & 0.316 & 2.40 & 4 & $8 \mathrm{H}$ & 0.4557 & 0.6979 \\
\hline 023762 & 0.323 & 0.23 & 5 & $10 \mathrm{H}$ & 0.4557 & 0.6979 \\
\hline 108927 & 0.316 & 2.40 & 6 & $12 \mathrm{H}$ & 0.4560 & 0.6978 \\
\hline 108914 & 0.315 & 4.50 & 6 & $12 \mathrm{R}$ & 0.4560 & 0.6978 \\
\hline 024266 & 0.321 & 0.08 & 6 & $12 \mathrm{R}$ & 0.4557 & 0.6979 \\
\hline 042014 & 0.325 & -1.1 & 6 & $12 \mathrm{R}$ & 0.4557 & 0.6978 \\
\hline 060186 & 0.316 & 2.40 & 6 & $12 \mathrm{R}$ & 0.4560 & 0.6979 \\
\hline 023763 & 0.322 & 0.08 & 7 & $14 \mathrm{H}$ & 0.4557 & 0.6979 \\
\hline 108906 & 0.316 & 2.40 & 9 & 18R1 & 0.4560 & 0.6990 \\
\hline 108913 & 0.306 & 6.50 & 9 & $18 \mathrm{R} 2$ & 0.4560 & 0.6200 \\
\hline 023764 & 0.323 & - & 10 & $20 \mathrm{H}$ & 0.4557 & 0.6979 \\
\hline 042510 & 0.316 & 2.40 & 12 & $24 \mathrm{R}$ & 0.4557 & 0.6979 \\
\hline 060328 & 0.316 & 2.40 & 15 & $30 \mathrm{H}$ & 0.4557 & 0.6979 \\
\hline 042511 & 0.316 & 2.50 & 18 & $36 \mathrm{R}$ & 0.4557 & 0.6979 \\
\hline
\end{tabular}

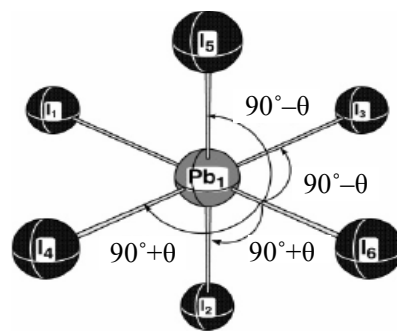

Figure 1. The near octahedral $\left[\mathrm{PbI}_{6}\right]^{4-}$. It has six equal $\mathrm{Pb}, \mathrm{I}$ distances, six I-Pb-I angles that are smaller than $90^{\circ}$ by $\theta$, and six 1-Pb-1 angles that are larger than $90^{\circ}$ by the same deviation. The angle $\theta$ is a measure of deviation from octahedral symmetry.

The actual mechanism of polytype formation in $\mathrm{PbI}_{2}$ is complex. There is still a great amount of dispute about the phase transformation even amongst the simple polytypes $2 \mathrm{H}, 12 \mathrm{R}$ and $4 \mathrm{H}$ which can co-exist in the same crystal in different proportions. Even higher order $\mathrm{PbI}_{2}$ polytypes could also exist in hexagonal or rhombohedral structure (Table 1). According to existing literature the structural defects, imperfections native/extrinsic impurities in $\mathrm{PbI}_{2}$ may provoke the co-existence of various polytypes. It is very difficult to detect any differrence between various polytypes by measuring physical properties which always reflects the average property of the material.

Earlier Chaudhary \& Kaur [9] have given a mechanism of phase transition between polytypes due to impurities. It is to be noted that the mechanism is only valid at a local scale in the vicinity of the impurity and the atoms rearrange within single layers. With this, it is difficult to explain the transformation of the whole crystal from one form to the other. The possible mechanism could be that transformation may spread over the whole crystal from a single nucleation centre or the whole structure may become unstable and atoms may become mobile enough to create a new stacking which is the most stable one.

\section{Studies on Storage of Lead Iodide Crystals}

$\mathrm{PbI}_{2}$ structure consists of various stackings of $\mathrm{PbI}_{2}$ sheets in each of which a layer of $\mathrm{Pb}$ ions is sandwiched between two close packed layers of iodine ions and I-Pb-I sandwich being the repeat unit. The binding within a sandwich believed to be largely ionic, is quite strong but two adjacent sandwiches are bound together with weak van der Walls forces.

As our interest goes for the device fabrication that may give reliable results for a long time, it becomes necessary to examine carefully the results of storage of $\mathrm{PbI}_{2}$ crystals (Table 2).

Out of seven categories there are four categories where the crystals do not transform to another structure after storage. 
Table 2. Results of X-ray characterization of $\mathrm{PbI}_{2}$ crystals and storage.

\begin{tabular}{|c|c|c|}
\hline No. of crystals & $\begin{array}{c}\text { Structure of as grown } \\
\text { crystals }\end{array}$ & Storage $^{*}$ \\
\hline \multicolumn{3}{|c|}{ 1) Crystals grown from gel (Soudmand \& Trigunayat (1989) [14]) } \\
\hline $\begin{array}{l}\text { Undoped crystals }(20) \\
40 \text { X-ray photographs }\end{array}$ & All 2H (40) & $\begin{array}{c}\text { After heating converted } \\
\text { to } 12 \mathrm{R} \text { but restored to } \\
2 \mathrm{H} \text { after storage }\end{array}$ \\
\hline $\begin{array}{l}\text { AgI doped crystals (15) } \\
30 \text { X-ray photographs }\end{array}$ & $\begin{array}{l}2 \mathrm{H}(21), 3 \mathrm{H}(3), 12 \mathrm{H} \\
(2), 16 \mathrm{H}(1), 12 \mathrm{R}(3)\end{array}$ & $\begin{array}{l}\text { Do not change to } 2 \mathrm{H} \\
\text { after heating and storing } \\
\text { at room temperature }\end{array}$ \\
\hline \multicolumn{3}{|c|}{ 2) Crystals grown from vapour (Jain \& Trigunayat (1996) [15]) } \\
\hline $\begin{array}{l}\text { Undoped crystals (20) } \\
40 \text { X-ray photographs }\end{array}$ & All 12R (40) & No change after storage \\
\hline $\begin{array}{l}\text { AgI doped crystals (20) } \\
40 \text { X-ray photographs }\end{array}$ & $\begin{array}{c}12 \mathrm{R}(13) \\
(4 \mathrm{H}+12 \mathrm{R})(16) \\
(2 \mathrm{H}+12 \mathrm{R})(11)\end{array}$ & $\begin{array}{l}\text { All crystals transform } \\
\text { to } 2 \mathrm{H}\end{array}$ \\
\hline \multicolumn{3}{|c|}{ 3) Crystals grown from melt (Chaudhary \& Trigunayat (1987) [16]) } \\
\hline $\begin{array}{l}\text { Very pure crystals } \\
39 \text { X-ray photographs } \\
(20 \text { zone passes })\end{array}$ & All 12R (39) & No change after storage \\
\hline $\begin{array}{c}\text { More pure crystals } 46 \\
\text { X-ray photographs } \\
\text { (12-14 zone passes) }\end{array}$ & $\begin{array}{c}12 \mathrm{R}(36) \\
4 \mathrm{H}+12 \mathrm{R}(8) \\
2 \mathrm{H}+12 \mathrm{R}(2)\end{array}$ & $\begin{array}{l}\text { All crystals transform } \\
\text { to } 2 \mathrm{H}\end{array}$ \\
\hline $\begin{array}{l}\text { Less pure crystals } \\
75 \mathrm{X} \text {-ray photographs } \\
(6-8 \text { zone passes })\end{array}$ & $\begin{array}{c}12 \mathrm{R}(51) \\
12 \mathrm{R}+4 \mathrm{H}(24)\end{array}$ & No change after storage \\
\hline
\end{tabular}

* Storage time in all the cases is few months.

1) Undoped crystals grown from gel (all $2 \mathrm{H}$ )

2) Undoped crystals grown from vapour (all $12 R$ )

3) Very pure crystals grown from melt (all $12 R$ )

4) Less pure crystals grown from melt (12R), $(12 R+$ $4 \mathrm{H})$

These observations clearly establish a link between purity of material and phase transitions between polytypes. The explanation for the choice of best crystals for detector is as follows.

In gel growth, the impurities can enter the crystals from both reactants and the gel medium whereas in vapour growth, comparatively pure crystals can be grown as only volatile impurities can enter into the crystal. Further streaking and arcing has frequently been reported in the gel grown crystals [7]. However in both the cases, size of the grown crystal is small. However, vapour grown crystals could be a better option for the devices.

When we examine crystals grown from melt, it is observed that structure of very pure and less pure crystals do not change after storage. If the degree of impurities is high, (less pure crystals) that may cause excessive distortion of octahedron, that makes the iodine planes uneven which leads to interlocking and blockage of iodine planes which in turn do not allow the various structures to transform to $2 \mathrm{H}$. In highly pure crystals that are deprived of impurities, there is no distortion of octahedron, thus no room temperature transformation may be expected. For the crystals containing intermediate amount of impurities ("more pure crystals" in Table 2) the iodine layers are not as close packed as should have been in absence of impurities. These layers are prone to translation/rotation due to weak binding and are responsible for the formation of polytypes. Wahab and Trigunayat have reported that such transformations can take place by layer displacements during crystal growth and high temperature treatment of polytypes $[10,11]$. The above observation indicates that phase transformation can also take place by suitable impurity and/or time effects. Though "less pure" crystals have not transformed after storage but it is expected that they may get transformed after a long period of storage. Thus less pure crystals should also be ignored for device fabrication.

Now we are left with two options, very pure crystals grown from melt and the crystals grown from vapour. Out of the two choices, it is very clear that material of melt grown crystals has been purified by zone refining technique and impurities present in the starting material like $\mathrm{Ag}$ and $\mathrm{Mn}$ have been reduced to $<<1 \mathrm{ppm}$ and crystal has grown as a perfect one. An X-ray diffraction showing the reflections of photographs $12 \mathrm{R}$ structure is reproduced in Figure 2. The photograph is free of streaking and arcing (that measure the degree of disorder and tilt boundaries in the crystal). Further in melt growth (using zone refining system) one can better manipulate the impurities if required, and have a control on the size of the crystal. Thus it is suggested that pure melt grown crystals are the best choice for the fabrication of the detectors as there is no change in structure during long storage and of course vapour grown crystals can be the next choice.

Further, the crystal growth improvements are required in order to reduce the structural defects and to increase electron/hole mobilities needed to improve the performance of lead iodide radiation detectors.

\section{Future Studies}

In most of the earlier studies made on $\mathrm{PbI}_{2}$ crystals, the role played by impurities and the vacancies was being ignored. The degree and the distribution of vacancies are also important parameters to be looked for the fabrication of devices from $\mathrm{PbI}_{2}$ crystals. It is expected that atoms

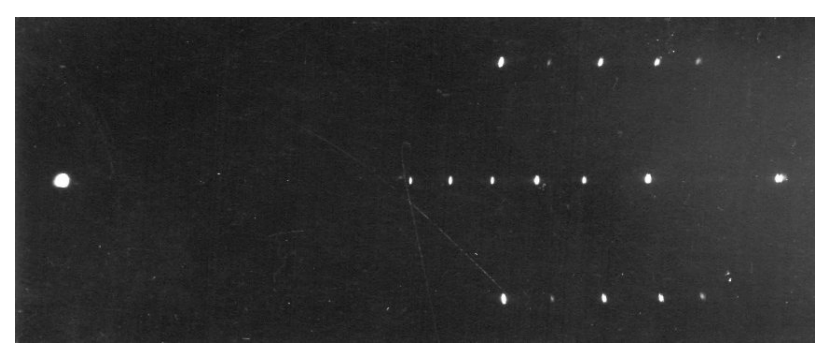

Figure 2. An a-axis $15^{\circ}$-oscillation photograph of $\mathrm{PbI}_{2}$ crystal showing the reflections of polytype $12 \mathrm{R}$. CuK $\alpha$ radiations; 3 cm camera. 
surrounded by a large number of vacancies should be considered to be more mobile than others along the layers. Further, the density of vacancies and their distribution play an important role in semi conducting properties of the materials. In a recent theoretical investigation Ito et al. [12] have calculated that for $\mathrm{SiC}$ polytype with $\mathrm{Si}$ vacancy, $6 \mathrm{H}$ structure is formed and $4 \mathrm{H}$ structure is favored in $\mathrm{C}$ vacancy condition. Thus showing that vacancies in $\mathrm{SiC}$ play an important role in stabilizing a particular structure. Similar calculations have been made on $\mathrm{ZnS}$ polytypic crystals [13].

\section{REFERENCES}

[1] M. Hassan, M. Matuchora and K. Zdansky, "Performance of Lead Iodide Nuclear Radiation Detectors with Introduction of Rare Earth Elements," Central European Journal of Physics, Vol. 4, No. 1, 2006, pp. 117-123. doi:10.1007/s11534-005-0010-X

[2] Y. M. Tairov and V. F. Tsvetkov, "Crystal Growth and Characterization of Polytypic Structures," Pergamon Press, Oxford, 1983.

[3] S. E. Saddow and A. Agarwal, "Advances in Silicon Carbide Processing and Applications," Artech House Inc., London, 2004.

[4] A. S. Raw, M. S. Furness, D. S. Gill, R. C. Adams, F. O. Holcombe Jr. and L. X. Yu, "Regulatory Considerations of Pharmaceutical Solid Polymorphism in Abbreviated new Drug Applications (ANDAs)," Advanced Drug Delivery Reviews, Vol. 56, No. 3, 2004, pp. 397-414. doi:10.1016/j.addr.2003.10.011

[5] M. Matuchoya, K. Zdansky, J. Zavdil, J. Tonn, M., Mousa, A.-G. Jafar, A. N. Danilewsky, A. Croll and J. Maixner, "Influence of Doping and Non-Stoichiometry on the Quality of Lead Iodide for Use in X-Ray Detection," Journal of Crystal Growth, Vol. 312, No. 8, 2010, pp. 1233-1239. doi:10.1016/j.jcrysgro.2009.12.034

[6] G. C. Trigunayat, "Present Status of Polytypism in $\mathrm{MX}_{2}$ Compounds," Phase Transitions: A Multinational Journal, Vol. 16-17, No. 1-4, 1989, pp. 509-527. doi: $10.1080 / 01411598908245726$
[7] E. Salje, B. Palosz and B. Wruck, "In Situ Observation of the Polytypic Phase Transition $2 \mathrm{H}-12 \mathrm{R}$ in $\mathrm{PbI}_{2}$ : Investigation of the Thermodynamic, Structural and Dielectric Properties," Journal of Physics C: Solid State Physics, Vol. 20, No. 26, 1987, pp. 4077- 4096.

[8] V. A. Bibik, I. V. Blonski, M. S. Brodin and N. A. Davydova, "Structural Phase Transition in Layer Semiconductor $\mathrm{PbI}_{2}$ Induced by Laser Irradiation," Physica Status Solidi (a), Vol. 90, No. 1, 1985, pp. K11-K14. doi:10.1002/pssa.2210900147

[9] S. K. Chaudhry and H. Kaur, "Impurity Induced Structural Phase Transformations in Melt Grown Single Crystals of Lead Iodide," Crystal Research and Technology, Vol. 46, No. 12, 2011, pp. 1235-1240. doi:10.1002/crat.201100250

[10] M. A. Wahab and G. C. Trigunayat, "Mode of Layer Displacements in $\mathrm{MX}_{2}$-Type Crystals," Solid State Communication, Vol. 36, No. 10, 1981, pp. 885-889. doi:10.1016/0038-1098(80)90133-7

[11] M. A. Wahab and G. C. Trigunayat, "Low Temperature X-Ray Diffraction Study of $\mathrm{CdI}_{2}$ Crystals," Crystal Research and Technology, Vol. 24, No. 4, 1989, pp. 355363. doi:10.1002/crat.2170240403

[12] T. Ito, T. Kondo, T. Akiyama and K. Nakamura, "Theoretical Investigation of the Polytypism in Silicon Carbide: Contribution of the Vacancy Formation," Physica Status Solidi (c), Vol. 8, No. 2, 2011, pp. 583-585. doi:10.1002/pssc. 201000401

[13] T. Ito, T. Kondo, T. Akiyama and K. Nakamura, "Theoretical Investigations for the Polytypism in Semiconductors," Journal of Crystal Growth, Vol. 318, No. 1, 2011, pp. 141-144. doi:10.1016/j.jcrysgro.2010.10.089

[14] M. Soudmand and G. C. Trigunayat, "Phase Transformations in $\mathrm{PbI}_{2}$ Crystals with Temperature," Phase Transitions, Vol. 16/17, 1989, pp. 417-424.

[15] A. Jain and G. C. Trigunayat, "Growth and Polytype Transformation of AgI-Doped and Undoped $\mathrm{PbI}_{2}$ Crystals," Acta Crystallographica Section A, Vol. A52, 1996, pp. 590-595.

[16] S. K. Chaudhary and G. C. Trigunayat, "Polytypism in Melt Grown Crystals of $\mathrm{CdI}_{2}, \mathrm{PbI}_{2}$ and $\mathrm{CdBr}_{2}$," Acta Crystallographica Section A, Vol. B43, 1987, pp. 225-230. 